\title{
Quaderni
}

QUADERNI Communication, technologies, pouvoir

\section{Une pratique collective de fabrication de l'autorité}

Jean-Claude Domenget et Antonin Segault

\section{(2) OpenEdition}

\section{Journals}

Édition électronique

URL : http://journals.openedition.org/quaderni/1082

DOI : 10.4000/quaderni.1082

ISSN : 2105-2956

\section{Éditeur}

Les éditions de la Maison des sciences de l'Homme

Édition imprimée

Date de publication : 5 mai 2017

Pagination : 85-97

Référence électronique

Jean-Claude Domenget et Antonin Segault, « Une pratique collective de fabrication de l'autorité », Quaderni [En ligne], 93 | Printemps 2017, mis en ligne le 05 mai 2019, consulté le 04 janvier 2020. URL: http://journals.openedition.org/quaderni/1082 ; DOI : 10.4000/quaderni.1082 


\section{Communication}

\section{Une pratique collective de fabrication de l'autorité}

Une étude de cas portant sur le live tweeting d'un événement professionnel au sein de la communauté des référenceurs a permis d'aborder le concept d'autorité dans sa dimension collective. Au-delà des questions "qui mentionne qui ? ", «qui retweete qui? » autant que "qui retweete quoi ? », existe-t-il une ambition de la part des acteurs de travailler l'autorité non seulement à un niveau individuel mais aussi au sein de collectifs? Dans ce cas, quels peuvent être les recoupements observables entre collectifs, groupes informels et publics dans les modalités de fabrication de l'autorité ? Une fois précisé le cadre théorique, nous présenterons la méthodologie construite afin d'analyser ces usages. À partir de là, différentes modalités de fabrication collective de l'autorité seront distinguées. Il

Maître de conférences en Sciences de l'information et de la communication Elliadd, Université de Bourgogne Franche-Comté

\section{Antonin Segault}

\section{Doctorant en Sciences de}

l'information et de la communication Elliadd, Université de Bourgogne Franche-Comté s'est avéré que le live tweeting dans un cadre professionnel est moins un moment de fabrication de l'autorité qu'un temps de reproduction de formes d'autorité existantes. S'appuyant peu sur des groupes au sein du réseau présents lors de l'événement, l'autorité se transmet à travers un mécanisme de circulation entre acteurs. Ces résultats, illustrant la dimension hybride des formes d'autorité cognitive, informationnelle et calculée ${ }^{1}$, sont discutés en fonction du type de collectif que constitue un groupe professionnel comme les référenceurs mais aussi, en fonction du niveau d'appropriation de Twitter par ces professionnels au sein de leurs trajectoires d'usage $^{2}$ de dispositifs de réseautage.

\section{Le live tweeting : un moment particulier de} fabrication de l'autorité

Dans le cadre d'un événement professionnel, le live tweeting peut être défini comme un partage 
de messages entre différents acteurs, par l'intermédiaire d'un mot-dièse ou hashtag, rassemblant une grande partie des échanges. Ce dispositif de communication intègre un collectif présent lors de l'événement et des publics, dont l'attention se focalise pendant cette période donnée sur les activités, les comportements, les discours de quelques acteurs, ayant le plus souvent un statut de célébrité et bénéficiant de formes d'autorité. Cette proposition de définition permet de préciser le cadre théorique dans lequel nous allons analyser cet usage de Twitter.

\section{La fabrication de l'autorité lors d'un événement professionnel}

En effet, Twitter peut être vu comme "une machine à fabriquer de l'autorité ${ }^{3} »$, au sein de laquelle « la propagation des tweets dépend ellemême d'une distribution complexe des degrés de légitimité, d'expertise et de notoriété des différents acteurs concernés. ${ }^{4} \gg$ Cette analyse prend tout son sens si l'on tient compte notamment de la dimension dissymétrique des interactions qui se déroulent dans ce dispositif entre d'un côté les « comptes élites ${ }^{6} »$ ou « micro-célébrités ${ }^{7}$ » et de l'autre les autres comptes. Le collectif constitué de pairs qui commentent l'événement trouve en Twitter un dispositif idéal dans lequel peut se mettre en place une communication d'influence ${ }^{8}$ entre une source d'autorité et ses publics.

Le live tweeting peut être analysé également comme un « cadre de référence ${ }^{9} »$ très codifié de fabrique de l'autorité, dont les caractéristiques ont été intériorisées par les différents acteurs en jeu. Les événements professionnels restent des rendez-vous au sein d'une profession (dans un lieu particulier et à une période précise de l'année) au cours desquels un professionnel peut chercher à se rendre visible, à se faire connaître s'il ne l'est pas encore, à entrer en contact avec un individu en particulier, à afficher son expertise dans un domaine spécifique, etc. et cela auprès de différents publics (pairs ou concurrents, clients ou prospects, médias). Se déroulant à la fois en présentiel et à distance via la médiation d'un dispositif sociotechnique comme Twitter, un tel événement est marqué du point de vue du régime attentionne ${ }^{10}$ par le basculement vers l'immersion. L'attention des participants se focalise sur l'échange en direct des messages et se détache du régime habituel de Twitter que constitue l'alerte ${ }^{11}$. Pour une majorité de participants, live tweeter ou suivre le fil de messages rassemblés autour d'un mot-dièse (hashtag) spécifique à l'événement sont devenus des actions routinières marquées par une recherche d'efficacité, à travers le maintien d'un équilibre entre une image d'authenticité qu'ils donnent d'eux et les attentes des publics imaginés $^{12}$.

\section{Trois formes d'autorité peuvent être distinguées}

Afin d'étudier les modalités de fabrication de l'autorité lors d'un tel événement, trois formes d'autorité peuvent être distinguées (l'autorité cognitive, l'autorité informationnelle et l'autorité calculée), en partant des travaux d'Évelyne Broudoux concernant la construction de l'autorité informationnelle sur le web ${ }^{13}$. Pratiquement synonyme de la notion d'influence choisie, l'autorité cognitive est dépendante de la crédibilité et de la réputation, deux critères de base de la création d'une confiance nécessaire à l'exercice d'une influence. Ensuite, « contrairement à l'autorité 
cognitive, l'autorité informationnelle, susceptible d'être portée par un individu ou un groupe, un objet, un outil cognitif ou encore un média n'a pas pour fonction principale l'influence mais celle d'in-former (donner une forme). ${ }^{14} \gg$ Étant donné que «le régime attentionnel de Twitter en fait surtout un vecteur d'autorité information$n^{n} l l e^{15} »$, il est alors intéressant de distinguer :

- l'autorité énonciative liée à la figure de l'auteur qui énonce. Elle peut être rapprochée du critère de la célébrité analysée par Nathalie Heinich ${ }^{16}$ et s'incarne notamment, dans le cas étudié, dans la figure du conférencier ;

- l'autorité institutionnelle ou groupe régulé par des règles hiérarchiques. Elle peut être recherchée dans les interactions autour du compte de l'association organisatrice de l'événement, voire de ses membres ;

- l'autorité de contenu qui concerne les types, la qualité et les sources des tweets. Elle permet de prendre en compte la diversité des contributions (information, partage de liens, conversation, commentaires, etc.).

Enfin troisième forme d'autorité, l'autorité calculée conduit à la définir comme une grandeur mesurable. «Affaire d'algorithme et d'interface, [...] cette autorité calculée se traduit en score que les plateformes et leur écosystème d'API se chargent de recenser, publier, comparer et exploiter. ${ }^{17} \gg$

Reste alors à préciser le type de collectifs au sein desquels se négocie la fabrication de l'autorité lors d'un tel événement. Rappelons que les collectifs d'usagers ${ }^{18}$ rassemblés sur Twitter ont été caractérisés comme des " petits mondes ${ }^{19}$ " marqués par l'homophilie, renouvelant la question de « ce qui fait lien ${ }^{20} »$. Lorsque le collectif est constitué de pairs appartenant pour la plupart à un même groupe professionnel ${ }^{21}$, comme dans le cas étudié, des interactions stables ${ }^{22}$ vont pouvoir être étudiées sous la forme d'une analyse de réseaux sociaux ${ }^{23}$.

\section{Analyser la fabrication de l'autorité sur Twitter}

Afin d'analyser la dimension collective de la fabrication de l'autorité lors d'un live tweeting, nous avons mixé différentes méthodes d'enquête : une observation participante, une analyse de réseaux sociaux et une analyse compréhensive des messages.

Une première méthode d'enquête a consisté à réaliser une observation participante lors de l'événement annuel de la communauté des référenceurs en France, le SEO Campus qui donne lieu chaque année à une série de conférences et d'ateliers sur des thématiques faisant l'actualité de la profession. S'étant tenu à Paris, les 13 et 14 mars 2014 pour une $6^{e}$ édition, rassemblant près de 400 personnes en présentiel et un nombre difficilement estimable à distance, via Twitter, cet événement réunit différents collectifs au sein de la profession (membres de l'association organisatrice, membres d'agence, membres de groupes informels tels que les black hats ${ }^{24}$, etc.).

Une seconde méthode a consisté à réaliser un recueil en direct puis a posteriori de tweets échangés lors de cet événement. Dans ce but, nous avons utilisé deux interfaces de Twitter, REST et Streaming. Parmi les très nombreuses 
fonctionnalités des APIs REST de Twitter, nous avons utilisé « GET search/tweets ${ }^{25}$ » pour collecter les tweets récents ${ }^{26}$ associés à un hashtag et «GET statuses/user_timeline ${ }^{27}$ » pour accéder aux derniers tweets d'un utilisateur. Afin d'obtenir un corpus plus complet autour d'un hashtag, nous avons également eu recours à la fonction «POST statuses/filter ${ }^{28}$ » de 1'API Streaming qui nécessite le maintien d'une connexion persistante, à travers laquelle sont envoyés les nouveaux tweets à mesure qu'ils sont créés. La veille de l'événement, qui s'est déroulé du jeudi 13 mars 2014 à 9 heures au vendredi 14 mars à 17h30, nous avons lancé la capture Streaming du hashtag \#seocampus. Nous avons par ailleurs utilisé « GET search/tweets » pour récupérer les tweets publiés avec ce même hashtag au cours des jours précédents (à partir du jeudi 6 mars). Le mardi 18 mars, quelques jours après l'événement, nous avons mis fin à la capture Streaming. Nous avons dans le même temps utilisé les APIs REST pour collecter les tweets de deux autres hashtags, \#seocamp et \#seocampus2014, ainsi que ceux émis par un groupe d'utilisateurs identifiés. Le corpus résultant de 4676 tweets est ainsi constitué à la fois de jeux de tweets exhaustifs et d'autres non-exhaustifs :

- \#seocampus : exhaustif du mercredi 12 mars (8:00) au mardi 18 mars (8:00), non exhaustif du jeudi 6 mars $(\sim 8: 25)$ au mercredi 12 mars (8:00);

- $\quad$ \#seocamp : non-exhaustif du mercredi (0:00) au lundi (23:59);

- \#seocampus2014 : non-exhaustif du mercredi (0:00) au lundi (23:59);

- \#utilisateurs : exhaustif du mercredi (0:00) au lundi (23:59).

Ces différents jeux de tweets comprennent ainsi :
- 805 tweets pour le jeu « seocampus2014 posteriori »;

- 158 tweets pour le jeu «seocampus_posteriori » ;

- 3713 tweets pour le jeu « seocampus streaming $»$.

L'observation participante et une connaissance approfondie de ce groupe professionnel et de sa structuration nous ont permis de distinguer 31 comptes $^{29}$ dont certains bénéficient d'une autorité cognitive. Il s'agit de comptes de conférenciers, de personnes de l'organisation (actuelle ou passée), de comptes «connus» dans la communauté SEO et de ceux d'agence. Le recueil a été réalisé a posteriori, suite à l'activité réalisée pendant l'événement mais il est exhaustif.

Ce recueil de données a permis de réaliser une analyse structurale de réseaux, en nous appuyant sur la théorie des graphes ${ }^{30}$. À côté des hashtags de l'événement, qui ont par définition une dimension collective, le critère principal de mesure de l'autorité que nous avons choisi est la mention, auquel nous avons associé parfois le marqueur de reprise, le retweet (RT). Ces marqueurs quantitatifs ont été complétés par une analyse compréhensive de quelques tweets afin d'analyser notamment les modalités de fabrication de l'autorité informationnelle.

\section{Les modalités de fabrication collectives de l'autorité}

Trois résultats principaux mettent en avant l'intérêt mais aussi les limites d'une analyse de la fabrication de l'autorité en termes de collectifs. 


\section{Une reproduction des formes d'autorité existantes}

Le live tweeting d'un événement professionnel est moins un moment de fabrication de l'autorité que de reproduction des autorités existantes. Il existe donc une certaine illusion d'une possibilité de gagner en autorité par une pratique de live tweeting pour un néophyte, dans un collectif tel que celui analysé. Cette reproduction s'illustre notamment par une prédominance de l'autorité énonciative comme critère des formes d'autorité informationnelles. Parmi les 10 comptes les plus mentionnés, 8 sont ceux de conférenciers. @ Seoblackhout (conférencier) a été mentionné 98 fois par 74 comptes différents. @Aleyda (conférencière) a été mentionné 92 fois par 50 comptes différents. Seuls@Miss_Seo_Girl, mentionné 57 fois par 49 comptes différents et @K_Lice, mentionné 46 fois par 41 comptes différents ne font pas partie cette année des conférenciers mais l'ont été les années précédentes.

Nous voulions ensuite vérifier si l'usage de mentions dans les tweets peut avoir comme visée de rendre visible son appartenance à un réseau et par là même de le renforcer. Nous avons analysé pour cela les tweets qui contiennent plus de 3 mentions, en intégrant les données du compte émetteur du tweet, du jour, de l'heure, du nombre de mentions et du texte du tweet. Nous avons pu constater que cette pratique de monstration du réseau a lieu aussi bien avant, pendant, qu'après l'événement : monstration du réseau par les absents avant, pour souhaiter aux présents un bon événement; tweets d'ambiance pendant, à travers la mise en scène de membres du réseau ou pratique d'un \#FF ( $\mathrm{Fol}$ low Friday) spécial événement « seocampus »; remerciements et auto-congratulations à visée publique après, etc. Par l'intermédiaire de ces messages, des groupes informels à dimension territoriale (ex : les Toulousains) ou thématique (ex : les black hats) sont mis en avant. Pourtant très peu de tweets s'appuient sur de tels réseaux pour asseoir l'autorité informationnelle (autorité de contenu) du message. Par exemple, le tweet : «@Yakiseo content de te rencontrer au \#seocampus prochain RDV Seocamp Albi \#SCD81 aveclestoulousains@Polo_Seo@Djibou_TeaM @GDTSB» met en avant un groupe informel à dimension territoriale. Celui-ci : «\#FF spécial \#seocampus :@abondance_com@Cariboo_seo@speyronnet@GPeyronnet@K_Lice @1YakaMama@Dixon_Jones@SimonGardette @ Seoblackout » propose un \#FF spécial événement. Tandis que ce dernier : "Nice pic dudes @Seoblackout@512banque@Missiz_ZRT (a)cubilizer: Aucune animosité entre BH/GG la preuve \#seocampus http://t.co/g50iOXyalL» évoque l'ambiance afin de mettre en avant un réseau thématique.

Autre résultat, les messages les plus repris rassemblent des critères d'autorité énonciative, d'autorité de contenu, voire d'autorité institutionnelle. Ce sont ceux de conférenciers (autorité énonciative) proposant un lien vers le support de leur présentation (autorité de contenu). Ce sont des tweets reprenant des phrases importantes entendues lors d'une conférence (autorité de contenu) mais aussi des messages d'ambiance, les deux étant renforcés par le type d'énonciateur (autorité énonciative, autorité institutionnelle). La forme des tweets (autorité de contenu) apparaît comme un critère important (au moins dans la représentation que s'en fait l'énonciateur) indi- 
quant une information inédite, la reprise d'une « petite phrase », le caractère inattendu d'une situation, etc. Pour illustration, les tweets suivants s'appuient sur des formes d'autorité énonciative (conférencier pour les deux premiers et microcélébrité au sein de ce groupe professionnel pour le troisième) et d'autorité de contenu (intégrant plus ou moins une dimension formelle) : «Le \#prezi de la conf \#SEO et \#Journalisme est disponible \#SEOcampus : http://t.co/sYHsxVGsJf Faites tourner si vous le souhaitez », "Le nouveau blog officiel Google France http://t. co/4T9WtUwMux \#seocampus http://t.co/ y9Az7sxAUY », «\#seocampus Scoop! Google annonce un blog français pour les webmasters. http://t.co/79OkXtgYGL \#referencement \#seo ». Tandis que le dernier intègre une dimension d'autorité institutionnelle (groupe informel) : «la selfie de l'année : 2 black hat seo et 2 googlers cc@Seoblackout@Missiz_Z\#seocampus \#seocampus2014 http://t.co/jT6GuMo45W».

\section{Les formes collectives d'autorité}

À côté de la dimension individuelle (et notamment du critère d'autorité énonciative), la dimension collective (correspondant aux critères d'autorité institutionnelle) n'apparaît que peu structurante dans la reproduction de l'autorité. Une analyse qualitative de graphes de mentions permet de relever cette dimension. Dans ce type de graphes, la taille des nœuds correspond à leur degré de centralité et l'épaisseur des arcs dépend du nombre de mentions. La réciprocité éventuelle des mentions est également prise en compte. Un algorithme de détection de « communautés » s'appuie sur les liens constitués par les mentions. Nous avons réalisé deux graphes [accessibles en ligne ${ }^{31}$ ], l'un « complet » pour avoir une vue d'ensemble du réseau de participants, l'autre « filtré » puisque seuls les arcs correspondant à plus de 3 mentions ont été représentés. Ce deuxième graphe permet de mieux distinguer les « grands » acteurs du réseau et les groupes auxquels ils sont reliés.

Ces graphes révèlent deux réseaux de « groupe » qui apparaissent distinctement au sein du réseau complet.

Le premier réseau s'organise autour de quatre conférenciers (@aleyda,@abondance_com, @LaurentPeyrat et@andrealpar). Il illustre clairement le peu d'importance de la dimension collective dans la reproduction de l'autorité. La cohésion de ce groupe est en effet faible en termes de densité de liens (nombre de liens observés en proportion de l'ensemble des liens possibles), même s'il présente une bonne connexité (liaisons directes et indirectes) avec d'autres comptes, de blogueurs, de spécialistes en communication événementielle, de membres d'agences partenaires, etc. (@Miss_Seo_Girl,@ValBdeMytte, @K_Lice, @lucmorineau). Par contre, la quasi absence de liens directs entre les comptes centraux de ce groupe (seul@aleyda mentionne @andrealpar) illustre le fait que ce dernier n'a pas d'existence en dehors de ces interactions en ligne.

Le second réseau s'appuie plus sur une dimension collective, puisqu'il s'organise autour du compte d'une agence partenaire @SYNODIANCE. Des liens de type bonding (liens augmentant la densité du réseau) viennent renforcer la cohésion du groupe autour du compte@yahn, associé de cette agence. Ce groupe est relié à d'autres usa- 
gers par des liens de type bridging (liens faibles connectant des composantes qui seraient autrement déconnectées du réseau) qui intègrent par exemple un autre conférencier (@YakaMama) à ce réseau, mais la distance (éloignement entre deux nœuds dans le réseau) entre ce membre et le groupe évoqué traduit la dimension éphémère de ce lien. Cet exemple illustre une recherche de renforcement d'autorité par la cohésion d'un groupe, mais celui-ci reste limité aux membres initiaux de ce dernier.

Le faible rôle de la dimension collective dans la reproduction d'autorité se retrouve également au sein des réseaux personnels.

Le réseau personnel autour du compte @Seoblackout est le plus important en termes de nombre de liens. Il occupe la position centrale dans le réseau complet, du fait de sa grande connexité. Par contre, l'appartenance de ce compte à un groupe informel (les Black Hats) et son rôle dans ce réseau hors ligne n'apparaissent pas du tout dans ce graphe de mentions. Ce résultat illustre le fait que ce groupe informel n'a pas besoin de se mentionner lors d'un tel événement pour se rendre visible.

Le réseau personnel autour du compte @renaudjoly est marqué par une forte intensité (fréquence élevée et diversité des membres le mentionnant) mais l'absence de réciprocité de la part de ce compte l'éloigne un peu du centre du réseau complet. C'est un compte qui est beaucoup mentionné mais qui mentionne peu.

Deux autres réseaux personnels sont marqués par une forte connexité (liens type bridging) mais varient en fonction de leur centralité dans le réseau complet. Celui autour du compte @RomainBellet présente une faible centralité car il est uniquement mentionné par les membres de son agence, ce qui constitue par ailleurs un sous-groupe thématique. Celui autour du compte @taiphan est plus central car il est mentionné par d'autres comptes également plus centraux. Ce type de réseaux personnels illustre la tactique de membres qui veulent jouer un rôle de connecteurs, mentionnant les comptes de conférenciers, d'organisateurs, de membres proches de l'organisation, etc.

Un dernier réseau personnel a attiré notre attention, celui autour du compte @lgalichet en raison de son isolement vis-à-vis du réseau complet. Les membres du réseau (à dimension territoriale) ont mentionné le compte d'un des leurs pour le rendre visible mais ils ont été les seuls à le faire.

Cette analyse de graphes de mentions ne permet pas de repérer de " cliques» (groupes au sein desquels la totalité des liens sont réciproques) lesquelles n'utilisent pas ce moyen pour montrer leur cohésion. Des échanges en face-à-face relèvent justement de l'intérêt de ce type d'événement en présentiel. De même, comme l'avait analysé Ronald S. Burt dans sa théorie des « trous structurau ${ }^{32} »$, l'asymétrie est une caractéristique essentielle des types d'interactions illustrées par des mentions. Les cas de réciprocité sont rares et se situent principalement au sein de groupes formels que constituent les agences ou l'association organisatrice de l'événement.

\section{Les formes de circulation de l'autorité}

Une circulation de l'autorité se met en place, 
partant de l'autorité énonciative du conférencier, bénéficiant parfois d'autorités institutionnelles, jouant sur l'autorité de contenu et visant l'autorité calculée. Pour illustrer ce phénomène, nous avons choisi d'analyser les échanges de messages lors de la conférence qui a suscité le plus de tweets, celle de Aleyda Solis, Head of Digital Strategy chez Woorank (outil d'évaluation SEO, e-marketing de sites web), intervenant sur le « local $S E O$ - référencement local ». Dès le début de la conférence, une concurrence apparaît entre comptes afin d'être le premier à relayer les idées fortes de la conférencière, tout en apportant éventuellement une plus-value (liens, commentaires, anecdotes, etc.). Pour donner un exemple, ces deux tweets de présentation de la conférence servent aux comptes qui souhaitent la relayer à se rendre visibles : «Le référencement local avec @aleydade@woorankau \#seocampus2014» http://t.co/fT6CRPflom [@web_Alliance à 14h12] et «Referencement local avec@Aleyda \#seocampus2014»http://t.co/lyhsGUSgwA [@Nat33_à 14h12]. Ainsi, un effet de silo, ou pour parler en termes $S E O$ de duplication de contenu, apparaît rapidement. Les mêmes messages ou presque sont diffusés à quelques secondes d'intervalle par plusieurs participants. Au cours de cette joute, des visuels illustrant le propos viennent souvent renforcer l'autorité de contenu du message. Par exemple: « \#seocampus2014 'Local SEO'by @aleyda from @Woorank-penser SOLOMO!http://t.co/ CfYSOFu9yg [@ValBdeMytteà 14h26]»est un message qui reprend une idée forte de la conférencière (autorité énonciative) en l'illustrant par une capture de la diapositive (autorité de contenu). D'autres tweets ont vocation à mettre en avant les connaissances ou compétences de l'usager, en s'appuyant également sur des formes d'autorité énonciative et de contenu. «Un outil (payant) sympa pour gérer son ref local (reporting, stats)http://t.co/R5sRxV7csjvia@aleyda \#seocampus» [@olek_tron à14h41]. De même certains tweets visent à bénéficier de l'autorité énonciative par circulation, comme celui-ci en fin de présentation : «@aleyda : one of the best and most complete speak of this \#seocampus Thank you!» [@cubilizer, à 14h54].

Tous les comptes qui suivent la conférence n'ont pas ce profil de relayeurs. Aussi, schématiquement, trois profils d'usagers se distinguent : les comptes qui relaient la conférence, apportent des commentaires, des ajouts, etc. ; ceux qui reprennent les premiers, enfin ceux qui suivent sans intervenir. Le jeu entre les deux premiers profils d'usagers conduit à la mise en place d'une autorité calculée, en fonction d'un algorithme intégrant une liste de critères dont le nombre de tweets, la fréquence de publication, le nombre de reprises (RT), et de citations, les sources des messages repris, etc.

Ces résultats, illustrant la dimension hybride des formes d'autorité cognitive, informationnelle et calculée, doivent être discutés en fonction du type de collectifs et du niveau d'appropriation du dispositif analysés.

\section{Reproduction de l'autorité et forme de collectifs}

Dans cette partie de discussion, nous souhaitons souligner que l'hypothèse d'une reproduction des formes d'autorité existantes n'est pas généralisable à tous les types de collectifs, et ne repré- 
sente pas une situation pérenne. Premièrement, les pratiques collectives analysées dépendent de « ce qui fait lien ${ }^{33}$ » entre les membres du groupe. Le réseau complet, analysé à partir des mentions entre ses membres, est un groupe professionnel ${ }^{34}$ car ces individus ont le même métier (référenceur SEO). Nous pouvons parler de " communauté » car des idées, valeurs, signes partagés établissent la particularité du groupe ${ }^{35}$. En termes d'analyse structurale de réseaux, ce groupe se caractérise par une connexité et une intensité élevées des relations entre les membres.

Deuxièmement, les usages de live tweeting analysés relèvent de pratiques de communication plus larges comme celles du réseautage. La notion de trajectoire d'usage, proposée par Serge Proulx et Guillaume Latzko-Toth ${ }^{36}$, permet justement d'étudier la continuité d'une pratique à travers les usages des divers dispositifs communicationnels qui se succèdent. Ainsi, le programme de ces chercheurs visait à «étudier les trajectoires d'usages individuels et collectifs, i.e. les parcours d'individus et de groupes (usages successifs et concomitants) à travers une constellation d'objets communicationnels passés, présents, émergents ou sur le déclin. ${ }^{37}$ » Dans cette lignée, les usages de live tweeting peuvent être analysés à la fois comme s'inscrivant dans un environnement normatif fait de patterns d'habitudes de réseautage et simultanément comme un usage créatif renouvelant les règles du réseautage. Certes, nous ne sommes plus dans une situation où les usagers sont majoritairement en phase d'appropriation du dispositif et de constitution de leur réseau relationnel. D'où ces résultats conduisant à conclure que le live tweeting est devenu moins un moment de fabrication (création) d'une autorité énonciative principalement qu'un moment de renforcement des formes d'autorité existantes (issue d'une présence construite). À l'inverse de nombreux facteurs conduisent à fragiliser cette reproduction (montée des incertitudes ${ }^{38}$, « crise » des identités professionnelles ${ }^{39}$, instabilité des dispositifs, etc.). Cette dynamique doit également être prise en compte à travers les changements apparaissant dans la fabrication des formes d'autorité informationnelle et calculée.

\section{Conclusion : changements et continuités dans la fabrication de l'autorité}

En guise de conclusion, les usages de live tweeting, au sein d'un groupe professionnel marqué par une grande cohésion, se caractérisent par une reproduction des formes d'autorités existantes. Ils s'appuient peu sur les groupes présents lors de l'événement et vont plutôt jouer sur un mécanisme de circulation de l'autorité entre acteurs. Ces résultats dépendent notamment du type de collectifs que constitue le groupe professionnel et du niveau d'appropriation du dispositif. Surtout, pour éviter de les surinterpréter et conduire à l'idée qu'un dispositif comme Twitter empêcherait toute évolution des positions au sein d'un réseau comme les référenceurs, quelques précautions méthodologiques s'imposent. Il s'agit notamment de resocialiser les données numériques ${ }^{40}$, en tenant compte de l'organisation des groupes formels ou informels auxquels les usagers appartiennent. Il faudrait étudier également si la population participant à l'événement évolue en termes de profils, d'une année à l'autre, ou s'il s'agit aujourd'hui d'un « rendez-vous entre amis ». De même, ces résultats indiquent que les relations révélées par les mentions ne 
sont pas forcément stables. Ainsi, l'analyse de ces usages nécessite de les intégrer dans une approche diachronique d'une pratique communicationnelle plus large, telle que le réseautage professionnel. L'exemple de l'évolution des enjeux du live tweeting montre les limites d'une analyse des pratiques autour d'un seul dispositif puisqu'une partie de l'échange se fait en dehors de celui-ci. S'inscrivant dans le programme des trajectoires d'usages entre dispositifs, ce type d'approche permet non seulement de tenir compte des patterns d'habitudes des usagers, mais aussi de révéler des espaces de créativité pour l'agir communicationnel.
$\mathrm{N} \cdot \mathrm{O} \cdot \mathrm{T} \cdot \mathrm{E} \cdot \mathrm{S}$

1. Éveline Broudoux, «Construction de l'autorité informationnelle sur le Web», 2007, disponible sur http://archivesic.ccsd.cnrs.fr/sic_00120710; Louise Merzeau, " Twitter: une machine à fabriquer de l'autorité », in Nicolas Pélissier et Gabriel Gallezot (dir.), Twitter. Un monde en tout petit?, Paris, L'Harmattan, 2013, pp. 35-51.

2. Serge Proulx, «Trajectoires d'usages des technologies de communication : les formes d'appropriation d'une culture numérique comme enjeu d'une « société du savoir " ", in Annales des télécommunications, vol. 57, n॰3-4, 2002, pp. 180-189.

3. Louise Merzeau, op. cit.

4. Ibid., pp. 37-38.

5. Jean-Claude Domenget, «De l'impératif de visibilité aux enjeux éthiques : les usages de Twitter par des professionnels du Web », in Serge Proulx, Mélanie Millette et Lorna Heaton (dir.), Médias sociaux : enjeux pour la communication, Québec, Presses de l’Université du Québec, 2012, pp. 217-232 ; Manuel Zacklad, «Twitter et ses multiples usages : la rançon du succès », in Nicolas Pélissier et Gabriel Gallezot (dir.), Twitter. Un monde en tout petit?, Paris, L'Harmattan, 2013, pp. 7-11.

6. Shaomei $\mathrm{Wu}$, Jacques $\mathrm{M}$. Hofman, Winter A. Mason et Duncan J. Watts, « Who says what to whom on Twitter ", in $W W W$ '11: Proceedings of the 20th International Conference on World Wide Web, 2011, pp. 705-714.

7. Alice E. Marwick et danah boyd «I tweet honestly, I Tweet passionately: Twitter users, context collapse and the imaginated audience », in New Media Society, vol. 13, no 1, 2010, pp. 114-133.

8. Thomas Beauvisage, Jean-Samuel Beuscart, 
Thomas Couronné et Kevin Mellet, « Le succès sur Internet repose-t-il sur la contagion ? Une analyse des recherches sur la viralité ", in Tracés. Revue de Sciences humaines, ${ }^{\circ} 21,2011$, disponible sur http:// traces.revues.org/5194

9. Patrice Flichy, L'innovation technique : récents développements en sciences sociales : vers une nouvelle théorie de l'innovation, Paris, La découverte, 1995.

10. Dominique Boullier, «Médiologie des régimes d'attention », in Yves Citton (dir.), L'économie de l'attention. Nouvel horizon du capitalisme ?, Paris, La Découverte, 2014, pp. 84-108.

11. Jean-Claude Domenget, «Le live tweeting. Formes d'autorité et régimes d'attention des publics », in Francine Charest, Alain Lavigne et Charles Moumouni (dir.), Médias sociaux et relations publiques, Québec, Presses de l'Université du Québec, 2015, pp. 83-99.

12. Alice E. Marwick et Danah Boyd (op. cit.) distinguent deux types d'usagers de Twitter, les gens ordinaires et les « micro-célébrités ». Les premiers désirent être authentiques, tandis que pour les seconds, l'authenticité n'est qu'un facteur de succès d'une stratégie visant à engager leur public. Dans ce but, ils révèlent quelques renseignements personnels, mentionnent des centres d'intérêts extérieurs, pertinents pour des publics variés. Ils cherchent surtout à maintenir un équilibre entre les dimensions personnelles et professionnelles dans leurs tweets. Pour les auteurs, cette méthode n'est pas sincère mais difficile à repérer. 13. Éveline Broudoux, op. cit.

14. Ibid., p. 5.

15. Louise Merzeau, op. cit., p. 43.

16. Nathalie Heinich, De la visibilité. Excellence et singularité en régime médiatique, Paris, Éditions Gallimard, 2012.

17. Louise Merzeau, op. cit., pp. 47-48.

18. Serge Proulx et Guillaume Latsko-Toth, « La virtualité comme catégorie pour penser le social: l'usage de la notion de communauté virtuelle ", in Sociologie et sociétés, vol. 32, no 2, 2000, pp. 99-122. 19. Nicolas Pélissier et Gabriel Gallezot (dir.), Twitter. Un monde en tout petit ?, Paris, L'Harmattan, 2013.

20. Serge Proulx, «Les communautés virtuelles : ce qui fait lien », in Serge Proulx, Louise Poissant et Michel Sénécal (dir.), Communautés virtuelles: penser et agir en réseau, Québec, Les Presses de l'Université Laval, 2006, pp. 13-25.

21. Claude Dubar, Pierre Tripier et Valérie Boussard, Sociologie des professions, Paris, Armand Colin, 3e édition, 2011 [1998].

22. Pour une distinction des notions de communauté et de réseau social permettant de caractériser des interactions sociales stables d'un côté et de l'autre les notions de foule et d'écume caractérisant des interactions beaucoup plus instables, voir Bernhard Rieder, « De la communauté à l'écume : quels concepts de sociabilité pour le « web social»? », in Tic\&Société, vol. $4, \mathrm{n}^{\circ} 1,2010$, disponible sur http://ticetsociete. revues.org/822

23. Alain Degenne et Michel Forsé. Les réseaux sociaux, Paris, Armand Colin, 2004 [1994].

24. Le sens du terme black hat en référencement diffère de celui utilisé en informatique. Il est employé pour " décrire le comportement d'un référenceur qui utilise des techniques contraires aux guidelines de Google». http://fr.wikipedia.org/wiki/Black_hat Par opposition, les white hats sont ceux qui respectent ces normes. 25. https://dev.twitter.com/rest/reference/get/search/ tweets. Notons néanmoins que «GET search/tweets» ne retourne pas l'ensemble des tweets utilisant un certain hashtag mais seulement un échantillon dont les caractéristiques sont mal connues. Twitter indique ainsi que l'API de recherche est centrée sur la pertinence et non l'exhaustivité (the Search API is focused on relevance and not completeness).

26. De l'ordre d'une semaine. 
27. https://dev.twitter.com/rest/reference/get/statuses/ user_timeline

28. https://dev.twitter.com/streaming/reference/post/ statuses/filter

29. Voici la liste des 31 comptes suivis : @taiphan, @aurelienbardon,@YakaMama,@MagicYoyo, @axenetvit,@clip6,@eseoref,@korleon38, @diije,@SYNODIANCE,@RaphSEO,@renaudjoly, @Miss_SEO_Girl,@Papilouve, @Seoblackhat, @1argow,@abondance_com,@RomainBellet, @mlocalseo,@olek_tron,@Polo_Seo,@web_Alliance,@Djibou_TeaM,@HonePro,@1ere_position, @intuiti,@yahn,@xavfun,@lerouxantoine,@cubilizer,@francoisgoube.

30. Alain Degenne et Michel Forsé, op. cit. ; Pierre Mercklé, Sociologie des réseaux sociaux, Paris, La Découverte, 2011 [2004].

31. Ces deux graphes peuvent être consultés en suivant ce lien : https://www.slideshare.net/JCDblog/ graphes-complet-et-filtr-de-mentions-article-domenget-et-segault-2017

32. Ronald S. Burt, " Le capital social, les trous structuraux et l'entrepreneur », in Revue française de sociologie, vol. XXXVI, n4, 1995, pp. 599-628.

33. Serge Proulx, «Les communautés virtuelles : ce qui fait lien », in Serge Proulx, Michel Sénécal et Louise Poissant (dir.), Communautés virtuelles : penser et agir en réseau, Québec, Presses de l'Université Laval, 2006, pp. 13-25.

34. Claude Dubar, Pierre Tripier et Valérie Boussard, op. cit.

35. Serge Proulx et Guillaume Latsko-Toth, op. cit. ; Bernhard Rieder, op. cit.

36. Serge Proulx, « Trajectoires d'usages des technologies de communication : les formes d'appropriation d'une culture numérique comme enjeu d'une « société du savoir » », in Annales des télécommunications, vol. 57, n³-4, 2002, pp. 180-189.
37. Ibid., p. 181.

38. Robert Castel, La montée des incertitudes. Travail, protection, statut de l'individu. Paris, Seuil, 2009.

39. Claude Dubar, La crise des identités. L'interprétation d'une mutation, Paris, PUF, 2010 (4e éd.).

40. Alan Ouakrat et Julien Mésangeau, « Resocialiser les traces d'activités numériques : une proposition qualitative pour les SIC », in Revue Française des Sciences de l'Information et de la Communication, $\mathrm{n}^{\circ} 8,2015$, disponible sur https://rfsic.revues.org/1795 


\section{$R \cdot E ́ \cdot S \cdot U \cdot M \cdot E ́$}

L'étude de cas présentée dans cet article, portant sur le live tweeting d'un événement professionnel, permet d'aborder le concept d'autorité dans sa dimension collective. À partir notamment d'une analyse structurale de réseaux, il ressort que cet usage de Twitter est moins un moment de fabrication de l'autorité, qu'un temps de reproduction de formes d'autorité existantes. Cette dernière est issue d'un mécanisme de circulation de l'autorité entre acteurs et s'appuie peu sur les groupes présents lors de l'événement. Ce résultat, illustrant la dimension hybride des formes d'autorité cognitive, informationnelle et calculée, est discuté dans un premier temps, en fonction du type de collectifs que constitue un groupe professionnel comme celui des référenceurs qui a été analysé et, dans un second temps, en fonction du niveau d'appropriation de Twitter par ces professionnels au sein de leurs « trajectoires d'usage » de dispositifs de réseautage.

\footnotetext{
Abstract

In this paper, we present a case study on the live tweeting of a professional event, in order to investigate the collective dimension of authority. Through our work, including social network analysis, we observe that this use of Twitter aims not so much to generate authority but rather to reproduce existing authority structures. This reproduction of authority comes from a process of authority flow between actors, and does not rely much on the groups attending the event. This result emphasizes the hybrid dimension - cognitive, informational, calculated of authority. We will firstly discuss it in relation to the type of communities - SEO experts - that were analysed and, then, according to the appropriation of Twitter that these professionals developed throughout their use of social networking websites.
} 
